\title{
Nanoscale
}

D) Check for updates

Cite this: Nanoscale, 2017, 9, 12409

\section{A one step method for the functional and property modification of DOPA based nanocoatings $\uparrow$}

\author{
Qinghua Lyu, ${ }^{a}$ Jieyu Zhang, ${ }^{b}$ Koon Gee Neoh (DD b and Christina Li Lin Chai (D *a
}

Biomimetic poly(catecholamine) coatings have gained much attention in recent years due to their versatility as functional materials. Despite this, only limited methods are available to modify the function and property of poly(catecholamine) coatings, primarily through post-modification methods. Our approach reported herein provides a simple approach to the fabrication of novel functionalized poly(catecholamine) coatings. The strategy employs the copolymerization of $\mathrm{N}$-Ac-3,4-dihydroxyphenylalanine methyl ester (NADOPAMe) with nucleophilic additives, giving rise to nano-coatings on various surfaces including plastic, metal, glass and polymers. With the appropriate choice of nucleophilic additives, coatings with desired properties can be achieved. This is demonstrated through the fabrication of a redox responsive coating based on NADOPAMe with cysteamine as additive, which shows a concentration-dependent glutathione (GSH) responsive behavior. The ability to utilize this as a controlled release system is also demonstrated.

Received 20th July 2017,

Accepted 3rd August 2017

DOI: $10.1039 / c 7 n r 05293 f$

rsc.li/nanoscale

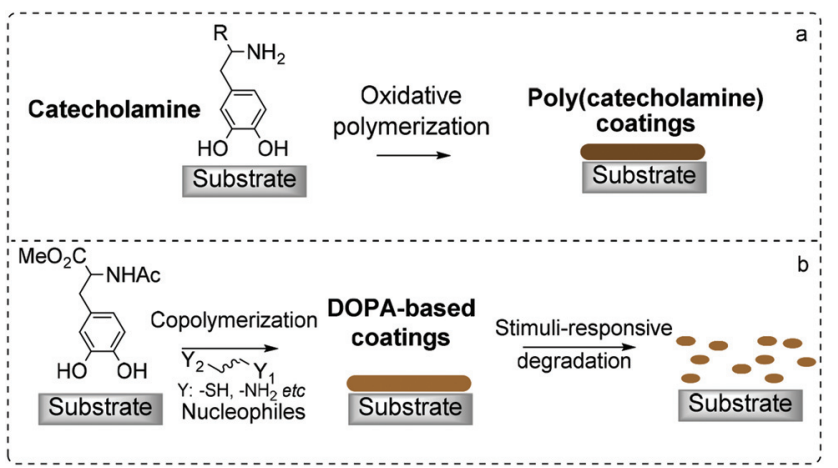

Scheme 1 A cartoon depiction of coatings based on catecholamines formed via (a) self-polymerization and (b) copolymerization strategy.

however, still controversial despite significant efforts to elucidate its structure. What is indisputable is that the formation of highly reactive quinone intermediates from catecholamines triggers the polymerization reaction.

The electrophilic quinone intermediates are not only susceptible to nucleophilic attack from the free amine of catecholamine monomers, but also to external nucleophiles. ${ }^{12,13}$ Nucleophiles such as thiols and amines can undergo Michaeltype addition and/or condensation reactions, respectively, with quinones. This is observed in some biomaterials, e.g. catechol-thiol conjugates in pheomelanin,${ }^{14}$ histidinyl-dopamine conjugates in insect exoskeleton, ${ }^{15}$ and lysyl-dopa residues in Mytilus edulis foot protein- $1{ }^{16}$ The variety of structures and functions of such biomaterials provide a significant opportu- 
nity in the development of new biomimetic functional materials. Surprisingly, there are only limited reports on the use of such a strategy in the development of new materials. $^{9,17-21}$

Our interest is in the use of 3,4-dihydroxyphenylalanine (DOPA) derivatives as monomers to form coatings on substrates. In this study, $\mathrm{N}$-Ac methyl ester of DOPA (NADOPAMe) was used to prevent the formation of complex eumelanin-like polymers, ${ }^{22}$ so that, the formed polymers may have more welldefined compositions compared to that of PDA. Nucleophilic additives were used for copolymerization reactions with NADOPAMe, and with the judicious choice of nucleophiles, stimuli-responsive coatings were fabricated (Scheme 1b).

\section{Results and discussion}

\section{Coatings from copolymerization of NADOPAMe and various different nucleophiles}

NADOPAMe was synthesized following the reported method (Scheme $\mathrm{S} 1 \dagger$ ), ${ }^{22}$ and characterized by ${ }^{1} \mathrm{H}$ and ${ }^{13} \mathrm{C}$ nuclear magnetic resonance spectroscopy as shown in Fig. S1.† The original dip-and-coat method pioneered by Messersmith et al. . $^{3,4}$ was employed in coating studies with NADOPAMe as monomer using silicon wafer as substrate. In contrast to studies with L-DOPA as monomer, ${ }^{4}$ no coating was formed on the silicon wafer when NADOPAMe was used (Fig. S2a $\dagger$ ). This is consistent with reported studies that the self-polymerization of catechol cannot form good coatings in the absence of nucleophiles. ${ }^{5,9,23,24}$ The addition of nucleophilic additives may overcome the limitations in the use of NADOPAMe and other catechols as monomers. Thus, an amended protocol was developed (Fig. 1a) and a range of commercially available nucleophiles (Fig. 1b) was tested for their ability to form coatings with NADOPAMe on silicon wafer. Depending on the nature of the nucleophiles, cross-linking with the $o$-quinone of
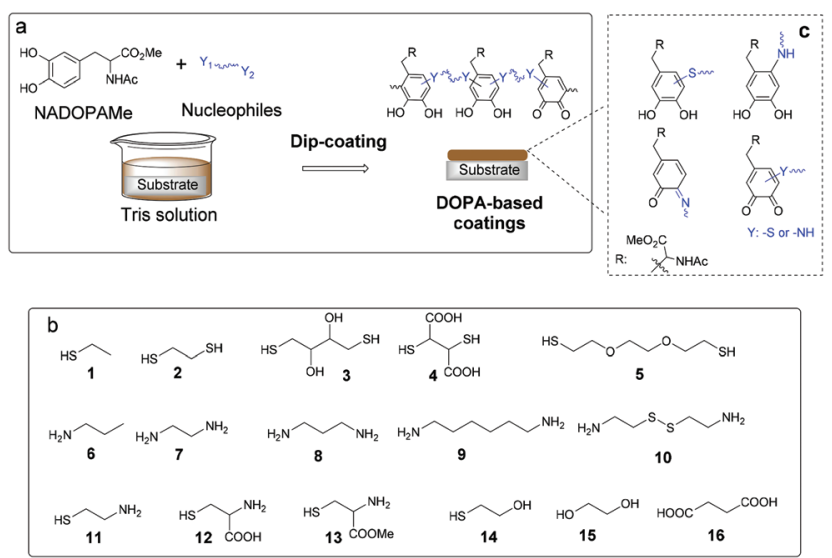

Fig. 1 (a) Schematic illustration of the protocol for the coating studies via copolymerization; (b) structures of nucleophiles selected for coating studies; (c) some structural units that may be present in NADOPAMenucleophile coatings.
NADOPAMe may occur via Michael addition and/or condensation reactions so as to bridge the catechol moieties in the construction of the coating materials (Fig. 1a and c). In these studies, NADOPAMe was first dissolved in tris buffer $(10 \mathrm{mM}$, $\mathrm{pH}$ 8.5) for 20 minutes. The silicon wafer was then immersed in the solution, followed by the addition of nucleophiles. The stoichiometry (1, 3, 5, 10 and 20 equivalents) of nucleophiles and the reaction times (24-72 h) were varied in order to assess their effects on coating formation. The ability to form coatings was examined visually, followed by AFM and XPS analyses wherever needed.

The choice of nucleophiles can be broadly categorized as sulfur (thiols 1-5), nitrogen (amines 6-10), bifunctional nucleophiles (11-14) and oxygen nucleophiles (alcohol 15 and carboxylic acid 16) (Fig. 1b). The copolymerization of monothiol 1 and monoamine 6 with NADOPAMe did not form any significant coating, while among the binucleophiles, the dithiols 2 and 5, the diamines 9 and 10, and cysteamine 11 formed coatings upon reaction with NADOPAMe. This suggests that a bifunctional nucleophile of appropriate length is minimally needed for cross-linking in the fabrication of coatings. The coatings that resulted from the use of the dithiols 2 and 5 (3 equivalents, tris buffer/methanol (2:1) mixture, 8-10 hours reaction time) were characterized using XPS, which showed the presence of sulfur $(164 \mathrm{eV})$ and nitrogen $(400 \mathrm{eV})$ on the surfaces (Fig. 2d and Fig. S3a $\dagger$ ). A minor sulfur peak (168 eV) was also observed and this may be attributed to the presence of oxidized sulfur. From AFM analyses of the samples NADOPAMe/2 (Fig. S2b $\dagger$ ) and NADOPAMe/5 (Fig. 2a), the surfaces were not uniformly coated, possibly due to the precipitation of disulfides arising from the competing oxidation of the thiols. Water-soluble thiols DTT 3 and DMSA 4 were used as nucleophiles to circumvent this problem, but no coating was observed.

The use of amines 6-8 (3 eq., tris buffer $\mathrm{pH} 8.5,72 \mathrm{~h}$ ) as nucleophiles did not lead to the formation of coatings. When the concentrations of amines 7 and $\mathbf{8}$ were increased to 20 equivalents ( $72 \mathrm{~h}$ ), large aggregates were observed on the surfaces, but these could be washed away with water during the washing step. In contrast, under similar conditions (20 eq., 56 hours), copolymerizing diamine 9 with NADOPAMe gave a thin, uneven coating of 8-10 nm (Fig. S2c $\dagger$ ), which was analyzed by XPS (Fig. S3b†). A thick light-yellow coating (30 nm) was obtained when the diamine $\mathbf{1 0}$ was used as nucleophile (3 eq., 36 hours) as shown in Fig. 2b. The use of cysteamine 11 as a bifunctional nucleophile with NADOPAMe led to the formation of a $25 \mathrm{~nm}$-thick yellow-brown coating after $48 \mathrm{~h}$ of copolymerization (Fig. 2c). XPS analysis (Fig. 2e) confirmed the presence of sulfur on the surface (binding energy of $164 \mathrm{eV}$ attributed to $\mathrm{S} 2 \mathrm{p}$ ). Due to the complex copolymerization process, the precise structure and/or components of the coating film remain unsolved. In addition to the intermolecular connections proposed in Fig. 1c, the copolymerization of NAODPAMe/11 may also undergo intramolecular cyclization to produce some benzothiazine-like intermediates (Scheme S2 $\dagger$ ), similar to the biosynthesis of pheomelanin. ${ }^{14,19}$ 

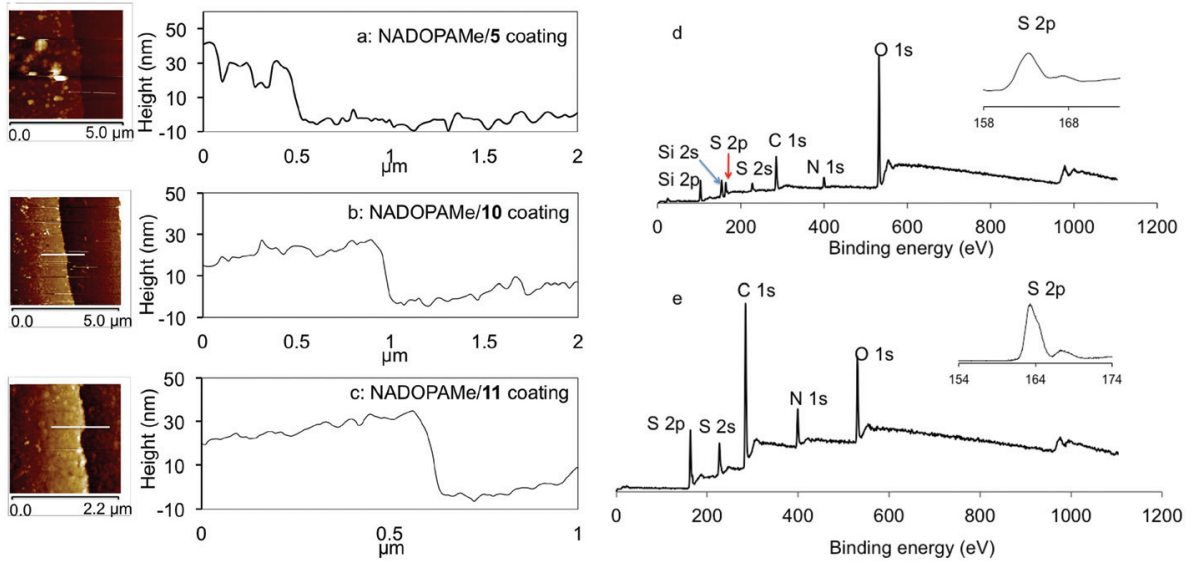

Fig. 2 (a-c) AFM analyses of coatings from copolymerization of dithiol 5 (3 eq., 18 h), diamine 10 (10 eq., 36 h) and cysteamine 11 (3 eq., 48 h) with NADOPAMe. (d \& e) XPS wide scan and S $2 p$ core level spectra for coatings of NADOPAMe/5 and NADOPAMe/11.

This successful formation of a coating with cysteamine $\mathbf{1 1}$ as compared to cysteine $\mathbf{1 2}$ and cysteine methyl ester $\mathbf{1 3}$ where no coating was formed suggests that the structure of the bifunctional nucleophiles is critical for coating formation. This may be related to steric and/or electronic effects of the nucleophilic entities.

To assess the versatility of the coating process on different substrates, glass, quartz, polystyrene, stainless steel and poly (lysine) were coated with the polymer of NADOPAMe/10 using the same protocol as outlined in Fig. 1. XPS characterization of these surfaces showed the presence of sulfur and nitrogen (Fig. S4 $\dagger$ ), which confirmed the successful coatings on these substrates. The water contact angles (CA) of all the coated substrates were measured. As shown in Fig. 3, the CA values of these substrates before coating varied from 20 to $85^{\circ}$, but after coating the CA values were consistently $49 \pm 3^{\circ}$. Similar experiments were carried out with NADOPAMe/11, in which the measured CA was $41 \pm 4^{\circ}$ on the different surfaces. These studies show that a variety of organic or inorganic surfaces can be modified using this copolymerization approach, similar to the universality of PDA surface modification.

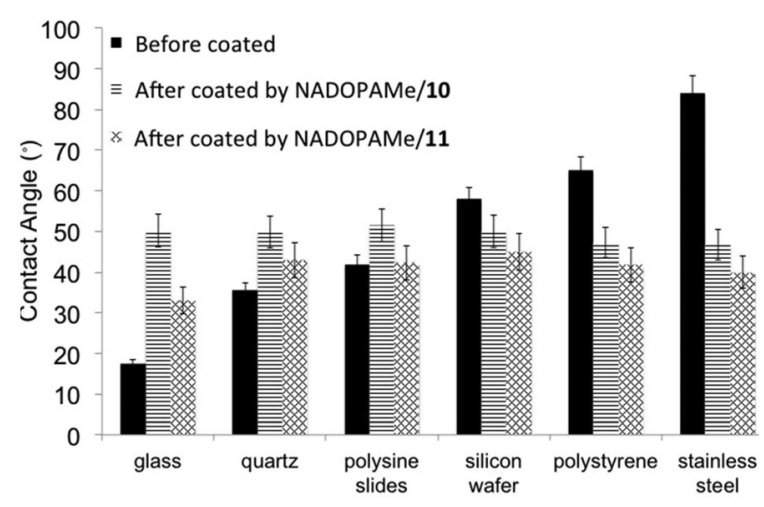

Fig. 3 Water contact angles of NADOPAMe/10 and NADOPAMe/11 coatings on different surfaces.
In order to gain some understanding into the structures of the oligomers/polymers that are involved in the formation of the coatings, attenuated total reflection-infrared spectroscopy (ATR-IR) and matrix assisted laser desorption ionization-time of flight mass spectrometry (MALDI-TOF MS) analysis of samples from the copolymerization of NADOPAMe with 2, 5, 9, 10 and 11, respectively, were carried out. As shown in Fig. 4, a broad band around $3400-3200 \mathrm{~cm}^{-1}$ region was assigned to $\mathrm{O}-\mathrm{H}$ and/or $\mathrm{N}-\mathrm{H}$ stretching vibrations of the catechol and/or amine groups. This IR absorbance is stronger for coatings formed using amines 9-11 than that with thiols 2 and 5, presumably due to the presence of free amine functionalities. The absorption bands around $3000-2850 \mathrm{~cm}^{-1}$ were assigned to the $\mathrm{C}-\mathrm{H}$ asymmetric and symmetric stretching vibration of $\mathrm{CH}_{2}$ aliphatic moiety. The dominant bands of $1760-1640 \mathrm{~cm}^{-1}$ were assigned to the $\mathrm{C}=\mathrm{O}$ vibrations of carboxylic ester (1735 $\left.\mathrm{cm}^{-1}\right)$, amide $\left(1625 \mathrm{~cm}^{-1}\right.$ ) and $o$-quinone functionality (1710-1665 $\mathrm{cm}^{-1}$ ), and the major absorbance at $1600-1510 \mathrm{~cm}^{-1}$ was ascribed to aromatic ring vibrations. Other vibrations in the 1450-1400 $\mathrm{cm}^{-1}$ region were associated with $\mathrm{CH}_{2}$ aliphatic vibrations from both NADOPAMe and the nucleophiles.

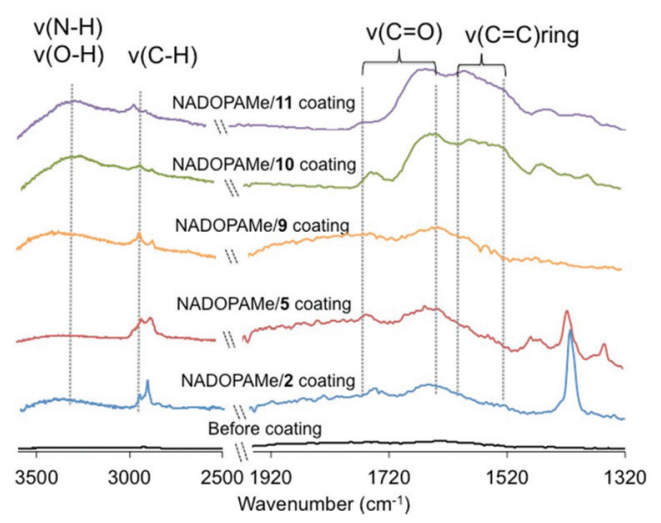

Fig. 4 ATR-FTIR spectra of copolymer coatings on poly(lysine) surfaces. 
The mass spectra of the copolymer samples (Fig. S5 and $6 \dagger$ ) were compared with that derived from the self-polymerization of NADOPAMe and DOPA methyl ester. Spectra of polyNADOPAMe showed a lower intensity of oligomeric composition as compared to that from the polymerization of DOPA methyl ester (Fig. S5†). This is consistent with the rationale that protection of the amino groups of DOPA will reduce competing reactions of intramolecular cyclization to form reactive dihydroxyindole intermediates and their subsequent polymerization. ${ }^{25}$ In general, the maximum $\mathrm{m} / \mathrm{z}$ values in the mass spectra of these copolymer coatings were less than 1000 (Fig. S6 $\dagger$ ). However, it should be noted that MALDI-TOF mass analysis is only able to provide information on oligomers that were dissolved in water/dimethyl formamide mixture, and not suitable for insoluble particles which may contain large oligomers. The common peaks observed in the spectra of the samples obtained from self-polymerization of NADOPAMe and its copolymers with nucleophiles suggest that the copolymerized coatings may contain some oligomers of NADOPAMe. For example, the $\mathrm{m} / \mathrm{z}$ value of $503.8 \mathrm{Da}$ is assigned to the dimer of NADOPAMe (Scheme S3†), which can arise from aryl-aryl coupling reaction. ${ }^{13,26-28}$

The above findings demonstrate the promise of using thiol linkers as additives in the construction of robust coatings with catecholamines. The interference of insoluble oxidized disulfides in aqueous solutions precludes the use of certain dithiol linkers but it is envisaged that these limitations can be over-

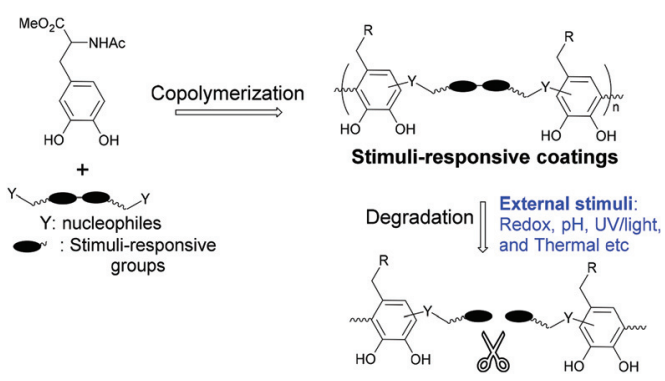

Scheme 2 Construction of stimuli-responsive coatings via copolymerization strategy. come with careful design of linker structures and control of reaction conditions. Amino nucleophiles have been shown to play a crucial role in the formation of stable coatings via cation $-\pi$ interaction during the self-polymerization of catecholamines or copolymerization with catechols. ${ }^{5,29}$ Our studies show that the length and the structure of the tether (e.g. 7-10) can affect non-covalent/covalent interactions between the oligomers formed and thus significantly influence the formation of the coatings. Our investigations also indicate that groups adjacent to the amino functionalities have a profound influence on the copolymerization process, as illustrated by the vastly different outcomes when cysteamine 11, cysteine 12 and cysteine methyl ester $\mathbf{1 3}$ were used as additives.

\section{Proof-concept study on the fabrication of redox-responsive coating}

Our methodology as described above can be applied to the fabrication of stimuli-responsive coatings in a simple and versatile manner, as outlined in Scheme 2. To demonstrate this, the redox responsive behavior of NADOPAMe/10 coating was studied. Glutathione (GSH) as reductant at concentrations of 1, 5, $20 \mathrm{mM}$ were chosen to provide the stimulus for studying degradation kinetics. ${ }^{30-32}$

Silicon wafer coated with NADOPAMe/10 was treated with $20 \mathrm{mM} \mathrm{GSH}$, and the degradation of the coating was monitored by XPS (Fig. 5a). The intensity of the disulfide signal, S 2p (164 eV), decreased with time of treatment while that of the substrate Si 2p (99.5 eV) increased with time. This suggests that the integrity of the film is compromised upon treatment with GSH. The degradation is also confirmed by FTIR analysis of NADOPAMe/10 coated poly(lysine) surfaces as shown in Fig. $5 \mathrm{~b}$. The IR peaks of the untreated coating observed at 3261 $(\nu(\mathrm{N}-\mathrm{H})$ and $\nu(\mathrm{O}-\mathrm{H})), 2951(\nu(\mathrm{C}-\mathrm{H})), 1735(\nu(\mathrm{C}=\mathrm{O}))$, and 1525 $\left(\nu(\mathrm{C}=\mathrm{C})_{\text {ring }}\right) \mathrm{cm}^{-1}$ were barely observable after treatment with GSH solution (20 mM, $4 \mathrm{~h})$. An analogous experiment was carried out to monitor coating degradation in situ using UV analysis (Fig. S7 and $8 \dagger$ ). In the presence of $1 \mathrm{mM} \mathrm{GSH} \mathrm{(8} \mathrm{h),}$ the absorbance at $380 \mathrm{~nm}$ showed a slight decrease but when the concentration of GSH increased to $5 \mathrm{mM}$, the absorbance at $380 \mathrm{~nm}$ decreased by approximately half in $4 \mathrm{~h}$. In the pres-
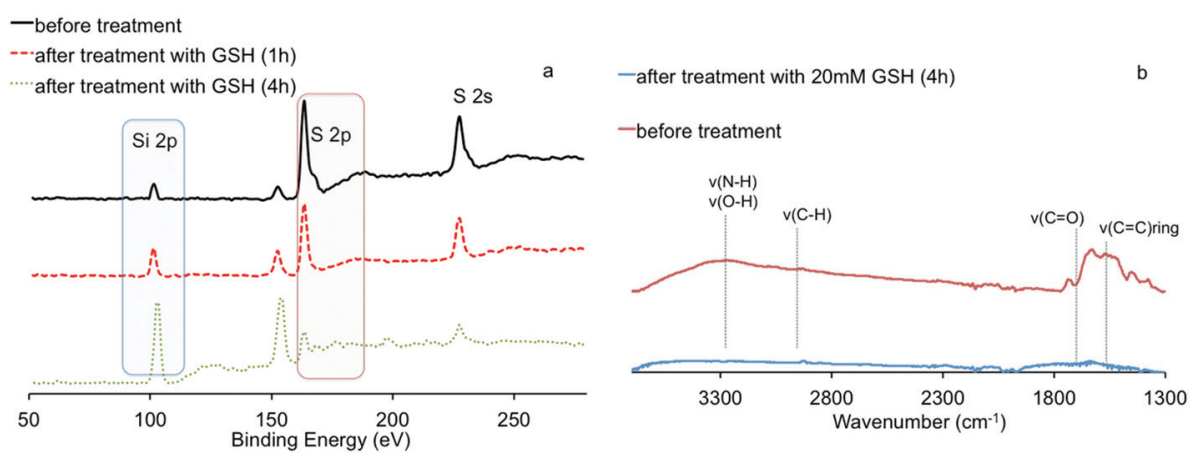

Fig. 5 (a) XPS spectra of NADOPAMe/10 on Si surface and (b) ATR-FTIR spectra of NADOPAMe/10 on poly(lysine) surface before and after treatment with $20 \mathrm{mM}$ GSH in tris buffer (10 mM, pH 7.4). 

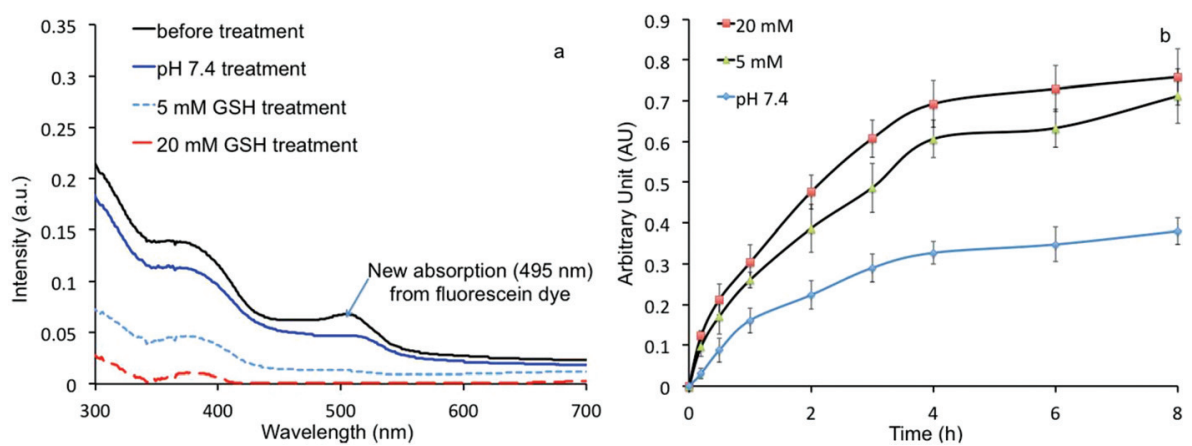

Fig. 6 (a) UV-vis absorbance of NADOPAMe/10/fluorescein on poly(lysine) before and after treatment with pH 7.4 tris buffer ( 8 h), $5 \mathrm{mM} \mathrm{GSH} \mathrm{(4} \mathrm{h)}$ or $20 \mathrm{mM} \mathrm{GSH} \mathrm{(4} \mathrm{h)} \mathrm{in} \mathrm{tris} \mathrm{buffer;} \mathrm{(b)} \mathrm{fluorescence-spectrometric} \mathrm{analyses} \mathrm{of} \mathrm{fluorescein} \mathrm{released} \mathrm{from} \mathrm{the} \mathrm{coatings} \mathrm{in} \mathrm{the} \mathrm{presence} \mathrm{of} \mathrm{different} \mathrm{con-}$ centrations of GSH.

ence of $20 \mathrm{mM}$ of GSH, almost all the coating was removed from the poly(lysine) surface by $4 \mathrm{~h}$. In a control experiment, the NADOPAMe/10 coating soaked in a $\mathrm{pH} 7.4$ tris buffer did not show significant changes over $8 \mathrm{~h}$ as indicated by XPS or UV analysis.

\section{Loading and release of test compound in redox-responsive coating}

To demonstrate that this stimuli-responsive coating film can be used as a release system, fluorescein dye was added together with the additive $\mathbf{1 0}$ to the tris solution of NADOPAMe following the same copolymerization protocol shown in Fig. 1. It is expected that fluorescein will be incorporated into the NADOPAMe/10 film when formed and will be released with the degradation of the coating upon GSH treatment. ${ }^{31}$ The incorporation of fluorescein into the coating was characterized by a new strong absorbance at $495 \mathrm{~nm}$ (Fig. 6a). The release kinetics of fluorescein upon GSH treatment was further monitored using fluorescence spectrometry. In the presence of $5 \mathrm{mM}$ or $20 \mathrm{mM}$ GSH solution, an initial fast release of fluorescein was observed due to the degradation of coatings and the cumulative release reached a plateau after $4 \mathrm{~h}$ (Fig. 6b). In contrast, the coating in $\mathrm{pH} 7.4$ buffer solution was stable over $8 \mathrm{~h}$, and the fluorescein was released at a much slower rate, presumably due to the swelling of the coating. These findings suggest that compounds can be successfully incorporated into these coatings via the copolymerization protocol, and their release can be triggered by appropriate stimuli, in our case, GSH as the reductant of the disulfide linkage.

\section{Conclusions}

In summary, we have developed a new strategy using nucleophilic additives to facilitate the polymerization of NADOPAME to form coatings. As demonstrated, stimuli-responsive coatings can be fabricated using this facile dip-and-coat process, which are potentially promising in applications such as release systems and for drug delivery. In contrast to poly(catechol- amine) coatings, where the choice of precursors is limited, the copolymerization of varied catechol(amine)s and functional additives can offer coatings with tailored chemical and physical properties. ${ }^{33,34}$ It is expected that this low-cost and facile bioinspired copolymerization approach will open new opportunities for the development of novel and versatile functional biomimetic materials for a range of applications.

\section{Experimental}

\section{Nucleophilic additives used for coating studies}

All the additives are commercially available and used without further purification: additives 1, 3-6, 9 and 12-14 were purchased from Sigma-Aldrich Pte Ltd; 2, 7, 8, 10 and 16 were purchased from Alfa Aesar; $\mathbf{1 1}$ and $\mathbf{1 5}$ were purchased from Tokyo Chemical Industry Co., Ltd (Singapore).

\section{Substrates used for coating studies}

Silicon (Si) wafers were obtained from Mitsubishi Silicon America, USA. AISI type 304 stainless steel foils $(0.05 \mathrm{~mm}$ in thickness) were purchased from Goodfellow Ltd of Cambridge, U.K., Quartz microscope slides (fused, $76.2 \mathrm{~mm} \times 25.4 \mathrm{~mm} \times$ $1.0 \mathrm{~mm}$ ) and glass slides $(76.2 \mathrm{~mm} \times 25.4 \mathrm{~mm}, 1-1.2 \mathrm{~mm}$ thick) were purchased from Alfa Aesar. Poly(lysine) slides (Thermo Scientific ${ }^{\mathrm{TM}} 25 \mathrm{~mm} \times 75 \mathrm{~mm} \times 1.0 \mathrm{~mm}$ ) and polystyrene dishes $(60 \mathrm{~mm} \times 15 \mathrm{~mm})$ were purchased from Fisher Scientific.

\section{General dip-and-coat method}

Si wafers were pre-cleaned with a solution of $4.8 \% \mathrm{HF}$ and $7 \%$ $\mathrm{HNO}_{3}$ in water, and then cleaned using ultrasound irradiation in deionized (DI) water and ethanol for $10 \mathrm{~min}$ each. Other substrates were first cleaned in an ultrasonic water bath for $30 \mathrm{~min}$, rinsed with ethanol, and then blown dry by nitrogen gas. The substrates, Si wafer $(1 \mathrm{~cm} \times 1 \mathrm{~cm})$, glass $(2.5 \mathrm{~cm} \times$ $2.5 \mathrm{~cm})$, poly(lysine) $(2.5 \mathrm{~cm} \times 2.5 \mathrm{~cm})$, stainless steel $(1 \mathrm{~cm} \times$ $1 \mathrm{~cm})$, polystyrene $(1 \mathrm{~cm} \times 1 \mathrm{~cm})$, quartz slides $(7.6 \mathrm{~cm} \times$ $2.5 \mathrm{~cm}$ ) were then immersed in $20 \mathrm{ml}$ of tris buffer $(10 \mathrm{mM}$, $\mathrm{pH}$ 8.5) with $10 \mathrm{mM}$ NADOPAMe for $20 \mathrm{~min}$, and additives 
were added at specified stoichiometries at room temperature. After coating for a specified time, the substrates were rinsed with water and ethanol, and dried by a stream of nitrogen gas. These samples were subsequently characterized as described below.

\section{Characterization of coatings}

Atomic force microscopy (AFM) analyses were carried out on Bruker Dimension ICON. X-ray photoelectron spectroscopy (XPS) measurements were performed on a Kratos AXIS Ultra HAS spectrometer with a monochromatized Al K $\alpha$ X-ray source (1486.6 eV photons), at a constant dwell time of $100 \mathrm{~ms}$ and pass energy of $40 \mathrm{eV}$. All binding energies were referenced to the $\mathrm{C}$ 1s hydrocarbon peak at $284.6 \mathrm{eV}$. Attenuated total reflection (ATR)-Fourier transform infrared spectroscopy (FTIR) analysis was carried out on a Bruker Alpha spectrometer equipped with (RT) DLaTGS detector (Bruker, USA) and a platinum ATR accessory with a $2 \mathrm{~mm}$ diameter single reflection diamond crystal. A clean silicon wafer and poly(lysine) slide were used as references respectively. Spectroscopic information was collected in the $1200-3600 \mathrm{~cm}^{-1}$ range with a $4 \mathrm{~cm}^{-1}$ resolution and 680 acquisitions per sample and corrected for baseline drift. Static water contact angles were measured using the sessile drop method with a $2 \mu \mathrm{L}$ water droplet in a telescopic goniometer (Rame-Hart Model 10000-(230)). Each static water contact angle reported was the mean value from three surfaces, with the value from each surface obtained by averaging the contact angles from at least three locations.

\section{Preparation of redox-responsive coating samples}

Different concentrations of GSH $(1,5$, or $20 \mathrm{mM})$ in tris solution (10 mM, pH 7.4) were prepared for the degradation study of NADOPAMe/10 film on Si wafer and poly(lysine) slides. Coated substrates, Si wafer $(1 \mathrm{~cm} \times 1 \mathrm{~cm})$ and poly(lysine) slides $(2.5 \mathrm{~cm} \times 2.5 \mathrm{~cm})$, were immersed in the above-mentioned GSH solutions or a control pH 7.4 buffer for a predetermined time, followed by washing with DI water and drying under nitrogen, and finally subjected to XPS, ATR-FTIR, and UV/Vis analysis. XPS and ATR-FTIR analysis of coated Si samples were carried out as described in the coating studies above. UV/Vis analysis of coated poly(lysine) samples were carried out using a Shimadzu UV-1800 UV-Visible spectrophotometer.

\section{Loading and release of fluorescein}

NADOPAMe/10 coatings with loaded fluorescein were prepared as follows: the pre-cleaned substrates, Si wafer $(1 \mathrm{~cm} \times 1 \mathrm{~cm})$ and poly(lysine) $(2.5 \mathrm{~cm} \times 2.5 \mathrm{~cm})$, were immersed in $20 \mathrm{ml}$ of tris buffer (10 mM, pH 8.5) with $10 \mathrm{mM}$ NADOPAMe for $20 \mathrm{~min}$, and then the nucleophile $10(30 \mathrm{mM})$ and fluorescein sodium salt $(5 \mathrm{mM})$ were added at room temperature. After 36-40 h, the substrates (Si wafer and poly(lysine)) slides were then taken out, washed with DI water and dried under nitrogen gas. The coated substrates were incubated in buffer solution (10 mM tris buffer, $\mathrm{pH} 7.4$ ) containing different concentrations of GSH (5, or $20 \mathrm{mM}$ ) as well as in blank tris buffer
(10 mM, pH 7.4). Time course measurements of the fluorescence intensity of these buffer solutions were carried out using a Hitachi F-7000 fluorescence spectrophotometer $\left(\lambda_{\text {ex }}=\right.$ $494 \mathrm{~nm} ; \lambda_{\mathrm{em}}=512 \mathrm{~nm}$ ) to compare the release kinetics of fluorescein under different GSH concentrations.

\section{Conflicts of interest}

There are no conflicts of interest to declare.

\section{Acknowledgements}

We thank SERC-A*STAR for financial support of this project (Project no: 1321202069).

\section{Notes and references}

1 Y. Liu, K. Ai and L. Lu, Chem. Rev., 2014, 114, 5057-5115.

2 M. Y. Liu, G. J. Zeng, K. Wang, Q. Wan, L. Tao, X. Y. Zhang and Y. Wei, Nanoscale, 2016, 8, 16819-16840.

3 H. Lee, S. M. Dellatore, W. M. Miller and P. B. Messersmith, Science, 2007, 318, 426-430.

4 J. H. Kuang, J. L. Guo and P. B. Messersmith, Adv. Mater. Interfaces, 2014, 1, 6.

5 C. Lim, J. Huang, S. Kim, H. Lee, H. Zeng and D. S. Hwang, Angew. Chem., Int. Ed., 2016, 55, 3342-3346.

6 S. M. Kang, J. Rho, I. S. Choi, P. B. Messersmith and H. Lee, J. Am. Chem. Soc., 2009, 131, 13224-13225.

7 R. Mrowczynski, R. Markiewicz and J. Liebscher, Polym. Int., 2016, 65, 1288-1299.

8 J. Liebscher, R. Mrowczynski, H. A. Scheidt, C. Filip, N. D. Hadade, R. Turcu, A. Bende and S. Beck, Langmuir, 2013, 29, 10539-10548.

9 S. Chen, X. Li, Z. L. Yang, S. Zhou, R. F. Luo, M. F. Maitz, Y. C. Zhao, J. Wang, K. Q. Xiong and N. Huang, Colloids Surf., B, 2014, 113, 125-133.

10 N. F. Della Vecchia, R. Avolio, M. Alfè, M. E. Errico, A. Napolitano and M. d'Ischia, Adv. Funct. Mater., 2013, 23, 1331-1340.

11 Y. H. Ding, L. T. Weng, M. Yang, Z. L. Yang, X. Lu, N. Huang and Y. Leng, Langmuir, 2014, 30, 12258-12269.

12 S. Bittner, Amino Acids, 2006, 30, 205-224.

13 J. Yang, M. A. C. Stuart and M. Kamperman, Chem. Soc. Rev., 2014, 43, 8271-8298.

14 L. Mosca, C. Foppoli, R. Coccia and M. A. Rosei, Pigm. Cell Res., 1996, 9, 117-125.

15 X. Huang, R. D. Xu, M. D. Hawley and K. J. Kramer, Bioorg. Chem., 1997, 25, 179-202.

16 S. X. Wang, N. Nakamura, M. Mure, J. P. Klinman and J. SandersLoehr, J. Biol. Chem., 1997, 272, 28841-28844.

17 X. N. Yin, J. Wang, J. J. Zhou and L. Li, Chin. J. Polym. Sci., 2015, 33, 1721-1729. 
18 Y. Lee, H. J. Chung, S. Yeo, C. H. Ahn, H. Lee, P. B. Messersmith and T. G. Park, Soft Matter, 2010, 6, 977983.

19 N. F. Della Vecchia, R. Marega, M. Ambrico, M. Iacomino, R. Micillo, A. Napolitano, D. Bonifazi and M. d'Ischia, J. Mater. Chem. C, 2015, 3, 6525-6531.

20 H. S. Kim, H. O. Ham, Y. J. Son, P. B. Messersmith and H. S. Yoo, J. Mater. Chem. B, 2013, 1, 3940-3949.

21 W. Z. Qiu, Y. Lv, Y. Du, H. C. Yang and Z. K. Xu, RSC Adv., 2016, 6, 34096-34102.

22 Y. S. Cheah, S. Santhanakrishnan, M. B. Sullivan, K. G. Neoh and C. L. L. Chai, Tetrahedron, 2016, 72, 6543-6550.

23 J. Saiz-Poseu, J. Sedo, B. Garcia, C. Benaiges, T. Parella, R. Alibes, J. Hernando, F. Busque and D. Ruiz-Molina, Adv. Mater., 2013, 25, 2066-2070.

24 Y. J. Liu, R. F. Luo, F. Y. Shen, L. L. Tang, J. Wang and N. Huang, Appl. Surf. Sci., 2015, 328, 163-169.

25 M. d'Ischia, A. Napolitano, V. Ball, C. T. Chen and M. J. Buehler, Acc. Chem. Res., 2014, 47, 3541-3550.
26 M. Yu, J. Hwang and T. J. Deming, J. Am. Chem. Soc., 1999, 121, 5825-5826.

27 L. M. McDowell, L. A. Burzio, J. H. Waite and J. Schaefer, J. Biol. Chem., 1999, 274, 20293-20295.

28 L. A. Burzio and J. H. Waite, Biochemistry, 2000, 39, 1114711153.

29 H. M. Hu, J. C. Dyke, B. A. Bowman, C. C. Ko and W. You, Langmuir, 2016, 32, 9873-9882.

30 A. Pompella and A. Corti, Front. Pharmacol., 2015, 6, 98.

31 D. Hong, H. Lee, B. J. Kim, T. Park, J. Y. Choi, M. Park, J. Lee, H. Cho, S. P. Hong, S. H. Yang, S. H. Jung, S. B. Ko and I. S. Choi, Nanoscale, 2015, 7, 20149-20154.

32 A. P. R. Johnston, G. K. Such and F. Caruso, Angew. Chem., Int. Ed., 2010, 49, 2664-2666.

33 E. Faure, C. Falentin-Daudre, C. Jerome, J. Lyskawa, D. Fournier, P. Woisel and C. Detrembleur, Prog. Polym. Sci., 2013, 38, 236-270.

34 J. Sedo, J. Saiz-Poseu, F. Busque and D. Ruiz-Molina, Adv. Mater., 2013, 25, 653-701. 